\title{
Invisible Work Meets Visible Work: Infrastructuring from the Perspective of Patients and Healthcare Professionals
}

\author{
Anna Sigridur Islind \\ Reykjavik University, \\ Department of \\ Computer Science, \\ Iceland \\ islind@ru.is \\ Helena Vallo Hult \\ University West / \\ NU Hospital \\ Group, Sweden \\ helena.vallo- \\ hult@hv.se
}

\author{
Victoria Johansson \\ University West, \\ Sweden \\ victoria.johansson \\ @,hv.se
}

\author{
Eva Angenete \\ University of \\ Gothenburg / \\ Sahlgrenska University \\ Hospital, Sweden \\ eva.angenete@gu.se
}

\author{
Martin Gellerstedt \\ Universtity of \\ Skövde, Sweden \\ $\underline{\text { martin.gellerstedt }}$ \\ a,his.se
}

\begin{abstract}
Increased patient engagement and the use of new types of data, such as patient-generated health data $(P G H D)$ is shifting how work is performed in relation to healthcare. This change enables healthcare professionals to delegate parts of work previously conducted by them to patients. There is a consensus regarding the need for nurses and physicians to work seamlessly together to make healthcare flow, but the role and responsibility of patients are less researched. In this paper, we aim to fill that gap by focusing on the shift of work from healthcare professionals to patients from the perspective of i) patients and ii) healthcare professionals. We use infrastructuring as a lens to understand the design of everyday work and actions from both perspectives. The main contribution is an analysis of, and insights into, how the work of patients can support healthcare professionals along with a conceptualization of how infrastructuring processes within and outside of healthcare are interconnected.
\end{abstract}

\section{Introduction}

The increased use of information technology (IT) to support healthcare is changing the prerequisites of the work performed by healthcare professionals [1]. This is especially true regarding IT that produces data, such as data that can be produced by the patient, and brought into the healthcare setting [2]. This changes the role of the patient since the patient can become increasingly aware and knowledgeable about their own condition. This type of shift thereby has the potential of allowing the patients to be more involved in their care, for instance, by self-tracking and engaging in peer discussions [3, 4]. Because patients can help each other and co-create their care through an increased responsibility in the care process; the tables are turning, and the patient becomes an increasingly important player in the future of healthcare [1]. Because of the increasing responsibility and involvement of the patient in healthcare in general, and in the formation of patient trajectories in particular, they can be perceived as one of the important stakeholder group that is contributing to the work within healthcare $[2,5]$. The changing role of the patient from passive consumer to an active contributor is one of the major shifts in modern healthcare. That particular shift is what this paper is about.

The fact that the patient has a more influential role in their own care also points to a gap in the literature regarding how the increased use of IT, and especially IT that produces data, affects the way work is shifted between the healthcare professionals and patients $[2,6]$. The way information technology can support the patient, help structure the patient-to-patient relationship and an understanding of the way patients perceive patient-to-healthcare exchange, has not been researched to the same extent as information technology supporting healthcare work [7]. Even less attention has been paid to the two-sidedness, and the two major roles, or stakeholder groups that outline healthcare interactions and how these two stakeholder groups, perceive the interplay with each other. These two stakeholder groups are: i) patients; ii) healthcare professionals.

Even though this increased power to the patient, and the changes in the role of the patient, and growing responsibility on the patient-side might solve the challenges healthcare is facing in relation to scarce resources, both globally and nationally, this change is not without contradictions. Due to the hierarchical nature of the patient to healthcare provider relationship, we have researched each of the stakeholder groups separately in prior studies, with focus on patients, nurses, and physicians respectively $[1,14,40]$.

In this paper, we provide an analysis of how patients that have had cancer, and healthcare professionals working with the care for chronically ill patients on a 
regular basis perceive their work through a multiple case study in Swedish healthcare. Furthermore, we analyze how these two stakeholder groups perceive their collaboration with the other stakeholder group through the theoretical lens of infrastructuring [8]. We refer broadly to infrastructuring as an activity which incorporates use, design and maintenance. The research questions are: (i) What type of infrastructuring do patients and healthcare professionals perform and; (ii) how does the infrastructuring of these heterogenous stakeholder groups intersect? The main contribution is an analysis of the design of everyday work and actions from the perspective of both stakeholder groups; insights into how patients' work can support the work of healthcare professionals and vice versa alongside a conceptualization of how the process of infrastructuring within and outside of healthcare are interconnected.

\section{Related Work}

Within the literature, there is a longstanding interest related to the digitalization of healthcare. The interest has moved from primarily focusing on the electronic health record (EHR), where research clustered around how the EHR affected work, towards an interest increasingly distributed work, with collaborative systems and how they affect work; that is now on the forefront of the research interest. Consequently, research efforts have historically often been focused on large-scale, national standards and strategic infrastructural changes with the aim of integrating various types of healthcare systems, where the EHR has been the leading challenge since the early 2000s [7, 9]. The interest in the EHR outlines an important aspect which stems from the notion that the healthcare sector, in general, has invested heavily in information systems, such as the EHR, as support for healthcare practices [7, 10-12]. However, as stressed earlier, the focus of research is shifting from the EHR as a working tool toward other types of information systems that can assist in different aspects of healthcare, such as collaboration and learning [13]. This include IT not only supporting healthcare professionals but with a dual, or triple aim of supporting different stakeholders within healthcare, while also supporting the stakeholder often missed; the patients. This shift and the growing interest in the complexity of the collaboration between the heterogeneous stakeholder groups in healthcare is what we would like to contribute to.

Evaluation of the condition at hand can be done through data, such as patient-generated health data (PGHD). PGHD encompasses data produced and collected by patients (or those seeking healthcare) and brought into healthcare for the purpose of enhancing the quality of care and pinpointing the problem at hand. It is a concept that does not only include data from wearables or similar sensors. Instead, PGHD includes qualitative self-assessment data as well, data that the patients can comprise, and bring into healthcare. The collection of PGHD, and engagement in self-monitoring through data can re-enforce the behaviors that prove to be of good value through visualizations of that data [2, 14]. The reasoning herein is, therefore, that not only do patients share data, and generate data, but they also learn through engagement with data, and through engagement with information from other patients. PGHD, as we see it can be one way to increase patient engagement but we also want to note that essentially, the increased patient engagement and use of new IT has in turn led to a significant growth in PGHD.

Through the use of such data, the patients can engage in their own care to a larger extent, with the dual purpose of triggering self-care for patients, and functioning as a decision-support for the healthcare professionals that help monitor that specific person [14], or in the long run, contribute to shared decision making.

Shared decision making (SDM) is a method to increase the patient's participation in the care process, which, in addition to shifts in everyday practices, also include shifts in role-relationships in healthcare. The method is based on the patient's right to be involved, informed, and take an active role in decisions concerning their health, care and support and make joint decisions about treatment. While there has been an agreement in arguing for a more equal and collaborative relationship and make shared decision making regarding the care process, the healthcare professionals' intentions to engage in shared decision-making is still uncertain [15]. In line with this, the concept of data work has been growing in interest. Data work in healthcare outlines work, supported by data, performed by both healthcare professionals and patients [16, 17]. When work is shifted from healthcare professionals, and when patients take on larger responsibilities, they also conduct work. The work includes engaging with informational support, understanding their own data, and informing others. The work is unpaid, but still can be considered work. What we would like to argue for, in this paper, in relation to shifts in data work, is that work cannot simply be moved; instead, the meaning has to be co-created through collaborations. The vast majority of the work on technological advances and collaboration work in healthcare, however, has focused on telecare performed as a part of call centers, and the care is viewed primarily in terms of the distribution of care, to a new place, or to new types of technological advancements [18-22]. There is however also research on healthcare contexts that shows that technology has slowly become an integrated part of care which in extension shows that 
technology has a bearing on the meaning of care, and has influenced both on the role of the professionals in healthcare, as well as on the patients' role, and data work [23]. Many studies on telecare or shifts in work from one stakeholder group to another are also before-and-after studies that rarely provide in-depth insights into the patients' side and the relation between the patient and healthcare professionals [24].

While the application of IT in healthcare, and data work, and increased impact of data on the meaning of care have become common areas of research interests in IS and related fields, the challenge of fully grasping the effects of how the patients' role can evolve, and enrichen modern healthcare remains. The increased responsibility of the patient, and the growing data flowing in healthcare, and the shifts in data work, is something that is changing the stakes of modern healthcare. As patients are increasingly becoming part of the data creation and have the possibility to be cocreators of the care trajectories and care processes alongside the healthcare professionals, it is increasingly important to understand the nature of these processes, including how those changes also trigger a chainreaction of changes that affect the nurses and physicians work, and how it affects the patients.

This paper does therefore not provide a before-andafter view, but instead look towards the processes of infrastructuring, i.e. the ongoing efforts by which i) patients design their own care and take part in data work; ii) healthcare professionals design their everyday tasks and iii) in what way that work intersects.

\section{Theoretical framing}

The term infrastructure originates from social studies of computing, where it was used as an analytical concept to study the sociology of technology, to denote the organizational and practical embeddedness of computing development, and the supporting resources that are often taken for granted [25, 26]. Star and Ruhleder [27] invoked the notion of 'infrastructure' an analytic framework to understand experience and failure. They argued for a reconceptualization of infrastructure - infrastructural inversion - as a way of foregrounding practices and conflicts of interest that might otherwise have remained unrecognized. Thus, extending the concept of infrastructure beyond a narrow view of infrastructures as large-scale technical installations, to include also political and social, potentially problematic, issues.

Pipek and Wulf [8] elaborate on Star and Ruhleder's [27] notion that infrastructure is a sociotechnical and relational concept. Their theory of infrastructuring is a framework for designing organizational information systems that focus on the role of IT as a work infrastructure, defined as: "the entirety of devices, tools, technologies, standards, conventions and protocols upon which the individual worker or the organization rely to carry out the tasks and achieve the goals assigned to them" [8, p. 455 ].

Infrastructures are not designed from scratch - but most developments methods emphasize design from scratch [8]. Information infrastructure is thereby not a stable entity but rather an ongoing social alignment between contexts. It is an enactment process, meaning that an infrastructure is constantly in the making and is something that emerges in practice [27-29]. When discussing infrastructure, there are three main types: 1) business sector infrastructure (e.g. infrastructure supporting the supply chain in car manufacturing), 2) universal service infrastructure (the internet) and 3) organizational corporate information infrastructure (complex integration of systems in an organization) [30, 31].

The infrastructure perspective has explanation power, particularly when it comes to existing infrastructure and how different systems relate to each other. However, when new infrastructures are being created and developed, infrastructuring as an extended perspective can be more useful [25]. Infrastructuring involves the process when infrastructures are being designed [8, 32]. Recently there is a renewed interest in health information infrastructures and the infrastructuring perspective [25, 33]. Infrastructuring can be particularly useful for the study of the design of work when both the universal service infrastructures and the organizational corporate information perspective are considered. Until now, limited attention has been paid to infrastructuring in healthcare, especially that include patients and caregivers [5]. When looking towards our case, where patients and healthcare professionals are designing their everyday work, the infrastructuring perspective can help shed light on how the work of the different stakeholders collides and intersects.

\section{Research approach}

This paper builds on qualitative data gathered in Sweden, involving both patients and healthcare professionals (nurses and physicians). The overall research approach for this paper is a multiple case study. The choice of conducting multiple case study can be described as a choice that rests on what object is to be studied, rather than merely a choice of techniques or methods [34]. Thus, it is more relevant to talk about a multiple case study in terms of an approach to study specific phenomena that can be seen in different cases, phenomena that are a part of a real-life context, or reallife contexts, which outlines a specific aspect of the 
multiple case study approach [35]. Multiple case studies can be positivistic [35], interpretive [36], or critical [37]. The approach in this multiple case study is interpretive $[36,38]$ primarily grounded in the data derived from the three cases included, where we looked for contextual details of each case, and patterns within and across the three cases [39]. Data collection, cases and corresponding studies are specified in Table 1.

Table 1: Empirical data and related studies

\begin{tabular}{|l|l|l|}
\hline Case & Empirical data & Reference \\
\hline Patients & 3 focus groups $(n=14)$ & {$[40]$} \\
\hline Nurses & 2 workshops $(n=6)$ & {$[14]$} \\
\hline Physicians & 3 focus groups $(n=17)$ & {$[1]$} \\
\hline
\end{tabular}

This analytical approach in this paper is based on analyzing the empirical data using an abductive approach abductive approach [41], with the specific aim to look for, and distinguish, various activities that contribute to infrastructuring [8] and map them into dimentions. Here, the abductive nature refers to the interplay between the empirical data, realized through real-world problems (inductively obtained) in combination with influences from theory (deductively inferred) [42] by viewing "reality from the theoretical viewpoint or perspective" [43, p. 104]. The abductive nature has thereby involved shifting between inductive and deductive reasoning as a way to continuously revise, sharpen and re-formulate the research design [42, 43] through engagement with coding of the three empirical cases. In regards to the three cases included in this multiple case study, the first case includes patients, the second case includes nurses and the third case includes physicians (see table 1). The patients have had cancer and undergone treatment for the cancer (ethical approval obtained from the regional ethical board of Gothenburg, EPN 262-18). Most of them classify as in need of chronic care and are learning to manage their relationship with the healthcare providers, while also learning to cope with self-management and changed lifestyle. All instances related to infrastructuring work (i.e. the process of designing everyday work through IT, information and data) by patients, nurses and physicians were coded and categorized as dimensions of infrastructuring specific to healthcare from the patients' perspective (outside view) and the healthcare professionals (inside view). These are described and further discussed below with examples (illustrative quotes) from different perspectives. The specific focus in this paper, is on the shift of work from healthcare professionals to the patients, which was the unit of analysis during the abductive process. Thus, although the work of physicians and nurses differ in content, tasks and responsibilities, their views are clustered and presented herein in comparison and in contrast to the patient perspective.

\section{Findings}

This section first provides insights from the way patients navigate their patient trajectory and pathway through healthcare, followed by empirical data on how healthcare professionals navigate their role.

\subsection{Patient perspective}

The findings of the study relating to the patient perspective (outside in), illustrates the patients view of their own journey, peer-relationships with others and the patient and healthcare professional relationship, including extending information to others (next-of-kin). The dimensions are summarized in Table 2 with sample quotes and is further described below.

\subsubsection{The patients view on their own journey}

The patients described their diagnostic journey as a process from receiving diagnosis to how they currently live with the diagnosis in everyday life. A common denominator was that infrastructuring was differently shaped depending on the level of diagnosis, and during which phase of the diagnostic journey the patient were currently situated: newly diagnosed, during/after treatment or surgery, or less frequency of medical visits. Infrastructuring for the patients could be divided into two phases: Phase survival and phase of entering the new normal and accepting the new way of life. Between these two phases is the emergence of a third phase; an in-between phase that combines phase survival and everyday life. For example, information and support is needed for understanding consequences of the decisions made in everyday life, in relation to getting to know your own body again (e.g. effects and consequences of food choices).

The patients reflected on their journey and that they had matured since they received their diagnosis. In the first phase, the infrastructuring is based on the newly diagnostic patients focus on survival and handling questions from relatives and friends, and in the later phases on practical information related to everyday life. In all, as described in this dimension, the most important factor during all diagnostic phases for the emergence of infrastructuring were the receiving of information, and what individual need for type of information and support that patients wanted vs. what information patients got from healthcare professionals, and how well this information met the particular need and state of the patient. Medical information is 
important, but the patients also need tips in relation to everyday life, which can sometimes be better given by other patients, as described in the following dimension.

\subsubsection{Peer-relationship of patients}

Another kind of work conducted by patients relates to information seeking and sharing of experiences. The patients share information between each other during the focus groups that are of practical nature, based on their experience from everyday life. They address that the practical tips are not a part of the follow-up care in health care and highlight that there is a discrepancy between the high-level information that the patients receive and what the meaning of the information entails for their everyday life after CPC treatment (such as vacuuming and practicalities around using the stoma bag). The participating patients agreed that the sharing of tips was important, and the hands-on experiences were helpful.

The patients reflected upon their participation and sharing, and the general impression was that it was helpful to discuss and they were in need to share and learn from each other. Even though they have matured and learned, there are critical moments when there is a need for emotional support, as the trauma of the cancer can pop up at all hours of the day. The patients articulate a need for support from people that share similar experience. They have not received that support during their follow-up care. This can be described as infrastructuring work, as the patients articulate that they receive - or would appreciate - support from peers rather than healthcare around primarily: i) concrete practical experience-based tips and tricks and ii) emotional support which is most suited from a person that has been through the journey.

\section{Table 2: Identified dimensions of infrastructuring work by patients}

\begin{tabular}{l}
\hline $\begin{array}{l}\text { Dimension patients and their view on } \\
\text { their own journey }\end{array}$ \\
Depending on level and phase; \\
patients perform infrastructuring \\
work by adapting information \\
given by healthcare to their \\
particular need and state of \\
diagnosis.
\end{tabular}

diagnosis.

\section{Peer-relationship of patients}

Discrepancy between high-level

information given to patients and what the meaning of the information entails for their everyday life. Patients conduct infrastructuring through the sharing of experience-based tips and tricks and emotional support with peer patients.

The patients view on the patientprovider relationship and extending information to others

Infrastructuring relating to handling feelings of being alone, and prioritizing what to bring and discuss with healthcare professionals, and information support on how to discuss with family and next-of-kin Example quotes from the study participants "Save your life, with surgery? Cancer or sex?.When diagnosed, certain information is not important, then all you think about is surviving. But later you wish you would have taken in the information about things that were less important then, such as sexuality."

"[It is situational] I want more information to avoid the consequences. "... "I got a hernia after surgery...it limits what I can lift... It's a little hard to know..."

"I was planning my own funeral [laughs], felt almost like, but now three years later, then I can look back at myself and say 'oh how stupid I was."

"things like flying - you are entitled to have an extra carry-on bag with you on the flight if you have stoma... because the suitcase may disappear"

"You get a lot of information about the regular chemotherapy side-effects, you know things like hypersensitivity...the doctor could have said to you, 'be careful now when you are vacuuming' [because] I wanted to repress everything, and vacuuming is the best way to do it, and then I tore away the skin [on my hands]. "

“...people say time, everything heals with time - no, not so much the things that are buried deep down. And that can pop up when you least expect it. And that's what I mean with support from people who have been there, that has the experience in an uneducated way so to speak, from the non-medical perspective." "with the injections you were here every other week, then you had support... a physical person that you could talk to who cared for me."

"I had follow-up for 6 months and then, in a way, I felt quite lonely and abandoned... because you cannot call the contact nurse and whine all the time."

"And when it comes to information to relatives, it is important. For myself, I may google and so, and there's a lot that I do not tell, or hide just to protect others."

"I have learned that I need to get as much information as possible [that's my job as a patient] and not to miss anything just because the next one has not told me." 


\subsubsection{The patients view on the patient-provider relationship and extending information to others}

There is a need to talk to healthcare, which is apparent through different comments made by the patients and even reflected in the choices of treatment methods. The need is also expressed when it comes to acute symptoms that are related to the cancer or cancer treatment, in which cases the health care becomes the center of contact, as it should. Several patients also talk about the initial decisions that they made, in collaboration with healthcare professionals. Such decisions related to the surgery path chosen, the chemotherapy afterwards, or radiation.

The patients agree on the weight of having medical professionals to discuss treatment options with, and that the health care professionals are key when it comes to medical issues. The patients however also reflect on a being "left alone" by health care during the after-care and between treatments. The respect for the health care professionals time is apparent and the patients articulate a need for continued support from health care. They articulate that they have received support during their treatment that has been important but some point towards being "left alone" after the treatment period is over. The feeling of being "left alone" was handled in terms of infrastructuring, as illustrated by one patient for example selecting treatment alternative which offered increased contact with health care professionals. The need for support from health care professionals mostly extends to discussions regarding symptoms and treatment alternatives, where the patients also take on responsibility for not "wasting" the professionals time.

However, the patients expressed also a need for information support when it comes to next-of-kin. Information on how to discuss sexuality and changed bodily functions was, according to the patients, something they wanted more of. Extending the information to the next-of-kin, is a struggle which also requires infrastructuring. Some information can be hard to express, and some information can be forgotten. Support for discussions with next-of-kin, and information sorting, so that the patient would not have to do the sorting of information was lacking. That type of support was not available during the follow-up care and would be appreciated by the patients. This is a gray area of work in the intersection of patients and providers where the next-of-kin on the one hand could potentially be involved as a resource or mediator between patient and healthcare, and on the other hand support is needed from healthcare professionals to mediate the information between patients and their next-of-kin.

To sum up, the patient journey involves infrastructuring work, as the patients must navigate and find the various types of support needed, which can be offered by: i) other patients and that type of support can include practical tips (ranging from e.g. food tips to stoma tips) while there is also an articulated need for emotional support and self-recognition from people with shared experiences, ii) support from health care professionals to discuss medical aspects, e.g. which treatment is the best and what medical route should be taken; and iii) information support on how to discuss with next-of-kin and there is a lack of information that is tailored for next-of-kin.

This mix of streams of support, described by one patient as "support from both staff and from people who have been on the same trip" characterizes the need for mixed support as a part of the follow-up care after cancer.

\subsection{Healthcare professionals' perspective}

The findings of the study relating to the healthcare professional perspective (inside out), illustrates the healthcare professionals view and understanding of the integrity and need of the patients; toward online health information and peer-relationship of patients; and the patient and healthcare professional relationship, including extending information to others (next-of-kin). The dimensions are summarized in table 3 with sample quotes and is further described below.

\subsubsection{Understanding the patient and respecting the integrity and need of the patients}

The healthcare professionals daily work involves to transition between various tasks and technologies such as on the one hand medical information systems (patient records, drug information, prescriptions etc.) which are secure, and provided within healthcare and on the other hand public health information (e.g. online platforms available for both healthcare professionals and patients and social media) which are commonly used both for work purposes and for private life. Both the nurses and the physicians commented that the new opportunities, afforded by digital platforms and social media, may be beneficial for both patients and healthcare at large, while also raising concerns about security aspects. Healthcare is highly regulated, accentuated by recent legislations (GDPR), and the professionals are well aware of the risks and challenges involved related to patient confidentiality and privacy.

The participants also highlight risks around commercial interests with the use of health apps that patients bring, as well for professional use of social media such as Facebook. A common experience from the healthcare professionals' perspective is that patients prefer traditional ways of communication, (e.g. paper 
documents), for safety (confidentiality) reasons, because they believe that is the safer choice. But they reflected on that it is also a responsibility for healthcare to provide a secure information environment that the patients feel they can trust.

In sum, this dimension illustrates a skepticism from the professionals due to uncertainties about regulations and confidentiality, with focus on risks and challenges (patient privacy) for the patients. The infrastructuring work comprises compliance with healthcare regulations while making use of the potential of new IT and respecting the integrity and need of the patients.

Table 3: Identified dimensions of infrastructuring work by healthcare professionals

\begin{tabular}{|c|c|}
\hline & \\
\hline $\begin{array}{l}\text { Understanding the patient and } \\
\text { respecting the integrity and need of } \\
\text { the patients }\end{array}$ & $\begin{array}{l}\text { "We have provided poor information; we have not provided enough } \\
\text { information about it to make the patients feel secure [to trust the digital]" } \\
\text { (Physician). }\end{array}$ \\
\hline $\begin{array}{l}\text { ing use } \\
\text { ital } \\
\text { atient }\end{array}$ & $\begin{array}{l}\text { "When we share data, it needs to be through an encrypted connection due to } \\
\text { sensitivity in the data. Nothing [referring to digital tools] is just simple to } \\
\text { use in healthcare, and we cannot just use what is used in regular businesses, } \\
\text { the patient always needs to come first." (Nurse). }\end{array}$ \\
\hline $\begin{array}{l}\text { Attitudes toward online health } \\
\text { information and peer-relationship of } \\
\text { patients } \\
\text { Infrastructuring relates to shifting } \\
\text { boundaries of traditional healthcare } \\
\text { work, and adjusting to a new role of } \\
\text { patients that take an active role and } \\
\text { engage in various ways in their own } \\
\text { care process }\end{array}$ & $\begin{array}{l}\text { "If the patients help each other then that it is just great. They know practical } \\
\text { things that we maybe don't know about, like how to mix the [specific } \\
\text { medication] into some breakfast. And even though we know how to apply the } \\
\text { gel [also medication] we don't do it every day, they do it every day. So. they } \\
\text { can help each other in that way. And learn from each other." (Nurse, about } \\
\text { the patients sharing knowledge with each other). } \\
\text { "They just press here [points to a button in the mobile application] and it's } \\
\text { so nice that it's so easy to just report an activity [referring to documenting } \\
\text { toilet visits]. Then, before we meet, I can just look at the data, and see the } \\
\text { details on the activities." (Nurse, on the patient gathering patient-generated } \\
\text { health data and self-monitoring). }\end{array}$ \\
\hline $\begin{array}{l}\text { tes to } \\
\text { PGHD } \\
\text { nic } \\
\text { lone in } \\
\text { lel }\end{array}$ & $\begin{array}{l}\text { The nurse often goes out of the clinic, to an office that the nurses' share, to be } \\
\text { able to concentrate on this new part of her work [using digital tools to discuss } \\
\text { with patients]. This, [walking to a new location] makes the transition even } \\
\text { more complicated. She is not able to take that risk of leaving for a secluded } \\
\text { location in an understaffed clinical clinic with urgent patient matters. She is } \\
\text { not able to concentrate on providing quality online posts in the clinic. She } \\
\text { wants to inform more patients through digital tools, such as social media, but } \\
\text { she is trained in one-to-one communication through consultations. } \\
\text { "Sometimes this feels overwhelming" (From an observation notes combined } \\
\text { with details provided from a nurse on using digital tool to discuss with } \\
\text { patients). }\end{array}$ \\
\hline
\end{tabular}

\subsubsection{Attitudes toward online health information and peer-relationship of patients}

The participating nurses and physicians shared experiences of patient generated health data and online patient information. They talked about this from several perspectives, highlighting both benefits as well as challenges and potential risks with patients engaging with each other on digital forums. On the one hand it facilitates the consultation and may decrease the workload if standard questions and test results can be accessed online. Here, the professionals indicate that while patients can help and support each other, it is still healthcare that have the medical responsibility, and that it is problematic if too much responsibility is put on the patients, although it has become more difficult to draw a distinct line. On the other hand, there is also the other end of this spectrum, with patients that lack access to digital tools or ability to access online information, where the professionals 
express that healthcare have a responsibility to provide for this type of patients as well. They talked about knowing the patient, and meeting every patient at the right level, and they expressed concern about that patients may worry more than necessary if they search for information on their symptoms online and believe they have cancer or something.

In sum, in this dimension infrastructuring work relating to shifting boundaries of traditional healthcare work and adjusting to a new role of patients that take an active role and engage in various ways in their own care process.

5.2.3. Healthcare perspective on the patientprovider relationship and extending information to others

The relationship boundaries between the healthcare professionals and the patients are shifting as a result increased patient influence, mediated by new information technologies. But the healthcare professionals remarked that with the new role of patients, the boundaries of traditional roles and responsibilities within healthcare are changing as well. Challenges relate also to core professional values from working in healthcare where there is a long history of rooted professionalism and maintaining a high-quality relationship with the patients is highly important. As well, there is little time to take on added work tasks, for instance engaging in digital collaboration with patients and peers or contribute to online discussions, when there is always patients and care work to go to. It adds digital stress to handle new and changed tasks and responsibilities, especially when the responsibility to find the balance and prioritize is imposed upon the professionals.

In sum, infrastructuring activities takes place both in the clinic (collegial/peer support) and in the patient and healthcare professional interactions, while work is done in old and new practices in parallel.

\section{Discussion}

The work of the patients can be related to the shift towards an increased interest in patient-generated health data (PGHD). In this paper, PGHD encompasses data produced and collected by patients, brought into healthcare for the purpose of enhancing the quality of care by pinpointing the problem at hand through data or increased information, in line with [1, 14]. The shift can also be related to the struggle of dependency versus independency that patient within chronic care, and healthcare professionals working with patients that are chronically ill, are in [37].
As such, PGHD and self-monitoring through data, where the patients increasingly engage with their own data, and can help each other through peer-support can re-enforce and strengthen specific behaviors that show through trends in the data to be of good value. When using such data, each data entry is not an autonomous entity; rather, it is a part of an array in which data builds up over time to a larger data-set that can then be visualized, for the dual purpose of triggering self-care for patients, and functioning as a decision-support for the healthcare professionals that help monitor that specific person [14] and contribute to shared decision making. This particular change, to a better informed, participating patient that is an active partner in the care process through their own data and information; an increasingly informed patient, is the point of departure in our empirical data. Gathering PGHD therefore represents the underlying cause for the shifts in data work from healthcare to patients shown in this paper. Through our study, we extend the understanding of work as a concept to also include the unpaid, discretionary, and in some cases invisible data work of patients, which complements the work and infrastructuring of the healthcare professionals presented herein. We have described the work of patients and the healthcare professionals, aiming for a better understanding of shifts in both the relationships and everyday practices. We confirm and extend findings from prior studies, by comparing and contrasting the infrastructuring from the perspectives of patients, as well as the healthcare professionals.

The theoretical framing is based on the sociotechnical, practice-oriented perspective of infrastructuring $[8,27]$. This perspective is as we see it a useful way of conceptualizing ongoing changes in the patient and healthcare professionals' interactions from two main perspectives: 'inside-out' and 'outsidein', as it acknowledges that infrastructures tend to emerge, rather than being fully planned, and sheds light on the technical-social, individual-organizational and local-global dimensions of developing new work practices.

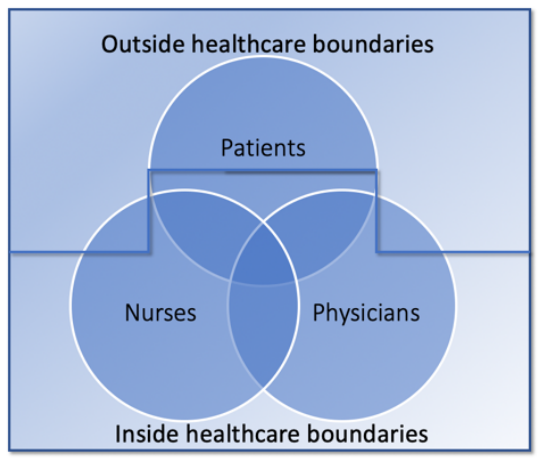

Figure 1: Infrastructuring in healthcare 
Figure 1 illustrates how the infrastructuring of the healthcare professionals on the one hand, and the patients on the other hand is intertwined. Additionally, we have found that the infrastructuring includes different levels of intertwined collaborations. Even though the figure illustrates a linear boundary.

Furthermore, the figure demonstrates the way we perceive the infrastructuring that is done outside healthcare boundaries as unpaid, discretionary, and invisible work, in most cases performed by the patients and the infrastructuring that takes place inside the boundaries of healthcare as structured, payed and visible work, performed in most cases performed by the healthcare professionals. What we have found in this paper, is that the infrastructuring done by the patients, supports the infrastructuring of the healthcare professionals and can therefore not be fully separated or disentangled.

\section{Conclusion}

In this study we highlight consequences and challenges related to the infrastructuring of patients and the healthcare professionals. Furthermore, we shed light on the invisible work of the patients. Faced with the challenges of working in complex and fragmented information environments, both the patients and the healthcare professionals described breakdowns and unintended consequences of using IT, PGHD and online health information when forming strategies and solutions to everyday practical problems; which we illustrate through their infrastructuring. The infrastructuring lens showed how patients on the one hand and healthcare professionals on the other hand perform collaborative infrastructuring to make use of increasingly complex information technologies entering healthcare, and how they use data (such as patient-generated health data), and discuss how they would like to utilize increased informational support both inside and outside of healthcare boundaries. From this paper we draw the conclusion that a central way of navigating healthcare, is to conduct infrastructuring within the stakeholder group (through peer-support between patients, and between healthcare professionals; nurses, and respectively physicians) while also showing the importance of working together between the stakeholder groups. Additionally, we shed light on how the infrastructures are intertwined, and what parts are not intertwined, but could potentially become, through increased integration between the stakeholder groups. Consequently, we show how the boundaries of healthcare are gradually expanding, and illustrate how healthcare work is changing, as a large proportion of healthcare work is now happening outside the boundaries of healthcare, through data work of the patients. Therefore, the invisible work of the patients and the changing role of the patients is a valuable asset to healthcare and in light of that, we suggest future research focuses on the patients' invisible work. Additionally, future research could dig deeper into how the invisible work differs and how increased invisible work shifts the boundaries between different professions within healthcare.

\section{References}

[1] H. Vallo Hult, A. Hansson, L. Svensson, and M. Gellerstedt, "Flipped healthcare for better or worse," Health Informatics Journal, vol. 25, no. 3, pp. 587-597, 2019.

[2] A. S. Islind, "Platformization: Co-Designing Digital Platforms in Practice," University West, 2018.

[3] D. Lupton, "The digitally engaged patient: Selfmonitoring and self-care in the digital health era," Social Theory \& Health, vol. 11, no. 3, pp. 256-270, 2013.

[4] G. Neff, and D. Nafus, The Self-Tracking: MIT Press, 2016.

[5] X. Gui, and Y. Chen, "Making Healthcare Infrastructure Work: Unpacking the Infrastructuring Work of Individuals." Proceedings of the 2019 CHI Conference on Human Factors in Computing Systems, pp. 1-14.

[6] L. Norström, A. S. Islind, and H. Vallo Hult, "Balancing the Social Media Seesaw in Public Sector: A Sociomaterial Perspective," IRIS Selected Papers of the Information Systems Research Seminar in Scandinavia, no. 8, 2017.

[7] G. Fitzpatrick, and G. Ellingsen, "A review of 25 years of CSCW research in healthcare: Contributions, challenges and future agendas," Computer Supported Cooperative Work: CSCW: An International Journal, vol. 22, no. 4-6, pp. 609-665, 2013.

[8] V. Pipek, and V. Wulf, "Infrastructuring: Toward an integrated perspective on the design and use of information technology," Journal of the Association of Information Systems, vol. 10, no. 5, pp. 447-473, 2009.

[9] E. Davidson, A. Baird, and K. Prince, "Opening the envelope of health care information systems research," Information and Organization, vol. 28, no. 3, pp. 140-151, 2018.

[10] C. LeRouge, V. Mantzana, and E. V. Wilson, "Healthcare information systems research, revelations and visions," European Journal of Information Systems, vol. 16, no. 6, pp. 669-671, 2007/12/01, 2007.

[11] M. Chiasson, M. Reddy, B. Kaplan, and E. Davidson, "Expanding multi-disciplinary approaches to healthcare information technologies: What does information systems offer medical informatics?," International journal of medical informatics, vol. 76, pp. S89-S97, 2007.

[12] C. Chantler, T. Clarke, and R. Granger, "Information technology in the English National Health Service," Jama, vol. 296, no. 18, pp. 2255-8, Nov 8, 2006.

[13] H. Vallo Hult, A. S. Islind, C. Östlund, H. Daniel, and P. Wekell, "Sociotechnical Co-design with General 
Pediatricians: Ripple Effects through Collaboration in Action," in Proceedings of the Twenty-sixth Americas Conference on Information Systems (AMCIS), 2020.

[14] A. S. Islind, T. Lindroth, J. Lundin, and G. Steineck, "Shift in translations: Data work with patient-generated health data in clinical practice," Health informatics journal, vol. 25, no. 3, pp. 577-586, 2019.

[15] F. Légaré, R. Adekpedjou, D. Stacey, S. Turcotte, J. Kryworuchko, I. D. Graham, A. Lyddiatt, M. C. Politi, R. Thomson, G. Elwyn, and et al., "Interventions for increasing the use of shared decision making by healthcare professionals," Cochrane Database of Systematic Reviews, no. 7,2018

[16] C. Bossen, K. H. Pine, F. Cabitza, G. Ellingsen, and E. M. Piras, "Data work in healthcare: An Introduction," Health Informatics Journal, vol. 25, no. 3, pp. 465-474, 2019/09/01, 2019.

[17] K. H. Pine, and C. Bossen, "Good organizational reasons for better medical records: The data work of clinical documentation integrity specialists," Big Data \& Society, vol. 7, no. 2, pp. 2053951720965616, 2020.

[18] R. Procter, J. Wherton, T. Greenhalgh, P. Sugarhood, M. Rouncefield, and S. Hinder, "Telecare Call Centre Work and Ageing in Place," Computer Supported Cooperative Work (CSCW), vol. 25, no. 1, pp. 79-105, 2016/02/01, 2016.

[19] C. Roberts, M. Mort, and C. Milligan, "Calling for Care:'Disembodied'work, teleoperators and older people living at home," Sociology, vol. 46, no. 3, pp. 490-506, 2012.

[20] C. Milligan, C. Roberts, and M. Mort, "Telecare and older people: who cares where?," Social science \& medicine, vol. 72, no. 3, pp. 347-354, 2011.

[21] J. Pols, and D. Willems, "Innovation and evaluation: taming and unleashing telecare technology," Sociology of health \& illness, vol. 33, no. 3, pp. 484-498, 2011.

[22] J. Pols, "The heart of the matter. About good nursing and telecare," Health care analysis, vol. 18, no. 4, pp. 374 388, 2010.

[23] A. S. Islind, and U. Lundh Snis, "From co-design to cocare: designing a collaborative practice in care," Systems, Signs \& Actions, vol. 11, no. 1, pp. 1-24, 2018.

[24] J. Kahn, "Virtual visits - confronting the challenges of telemedicine," N Engl J Med, vol. 372, no. 18, pp. 16841685, 2015.

[25] C. Lee, and K. Schmidt, "A Bridge too Far?: Critical Remarks on the Concept of "Infrastructure" in CSCW and IS," Socio-Informatics: A Practice-based Perspective on the Design and Use of IT Artifacts, V. Wulf, V. Pipek, D. Randall, M. Rohde, K. Schmidt and G. Stevens, eds., pp. 177-217, Oxford: Oxford University Press, 2018.

[26] R. Kling, "Defining the boundaries of computing across complex organizations," Critical issues in information systems research, pp. 307-362, 1987.

[27] S. L. Star, and K. Ruhleder, "Steps toward an ecology of infrastructure: Design and access for large information spaces," Information systems research, vol. 7, no. 1, pp. 111134, 1996.
[28] S. L. Star, "The ethnography of infrastructure," American behavioral scientist, vol. 43, no. 3, pp. 377-391, 1999.

[29] G. C. Bowker, K. Baker, F. Millerand, and D. Ribes, "Toward Information Infrastructure Studies: Ways of Knowing in a Networked Environment," International Handbook of Internet Research, J. Hunsinger, L. Klastrup and M. Allen, eds., pp. 97-117, Dordrecht: Springer Netherlands, 2010.

[30] O. Hanseth, and K. Lyytinen, "Theorizing about the design of Information Infrastructures: design kernel theories and principles," Sprouts: Working Papers on Information Systems, vol. 4, no. 12, 2004.

[31] M. Aanestad, M. Grisot, O. Hanseth, and P. Vassilakopoulou, "Information Infrastructures for eHealth," Information Infrastructures within European Health Care, pp. 11-23: Springer, 2017.

[32] E. Parmiggiani, "This Is Not a Fish: On the Scale and Politics of Infrastructure Design Studies," Computer Supported Cooperative Work (CSCW), vol. 26, no. 1-2, pp. 205-243, 2017.

[33] T. Greenhalgh, J. Wherton, S. Shaw, C. Papoutsi, S. Vijayaraghavan, and R. Stones, "Infrastructure Revisited: An Ethnographic Case Study of how Health Information Infrastructure Shapes and Constrains Technological Innovation," J Med Internet Res, vol. 21, no. 12, pp. e16093, 2019/12/19, 2019.

[34] R. E. Stake, The art of case study research: Sage, 1995.

[35] R. K. Yin, Case study research: Design and methods: Sage publications, 2013.

[36] G. Walsham, Interpreting information systems in organizations: John Wiley \& Sons, Inc., 1993.

[37] W. Carr, and S. Kemmis, Becoming critical: education knowledge and action research: Routledge, 2003.

[38] G. Walsham, "Doing interpretive research," European journal of information systems, vol. 15, no. 3, pp. 320-330, 2006.

[39] M. B. Miles, and A. M. Huberman, Qualitative data analysis: an expanded sourcebook, Thousand Oaks, CA: Sage, 1994.

[40] A. S. Islind, V. Johansson, H. Vallo Hult, P. Alsén, E. Andreasson, E. Angenete, and M. Gellerstedt, "Towards individualized blended care for patients with colorectal cancer: the patient's view on informational support," forthcoming.

[41] U. H. Graneheim, and B. Lundman, "Qualitative content analysis in nursing research: concepts, procedures and measures to achieve trustworthiness," Nurse Educ Today, vol. 24, no. 2, pp. 105-12, Feb, 2004.

[42] R. Gregory, and J. Muntermann, "Theorizing in design science research: inductive versus deductive approaches," 2011.

[43] A. H. Van de Ven, Engaged scholarship: A guide for organizational and social research: Oxford University Press on Demand, 2007. 